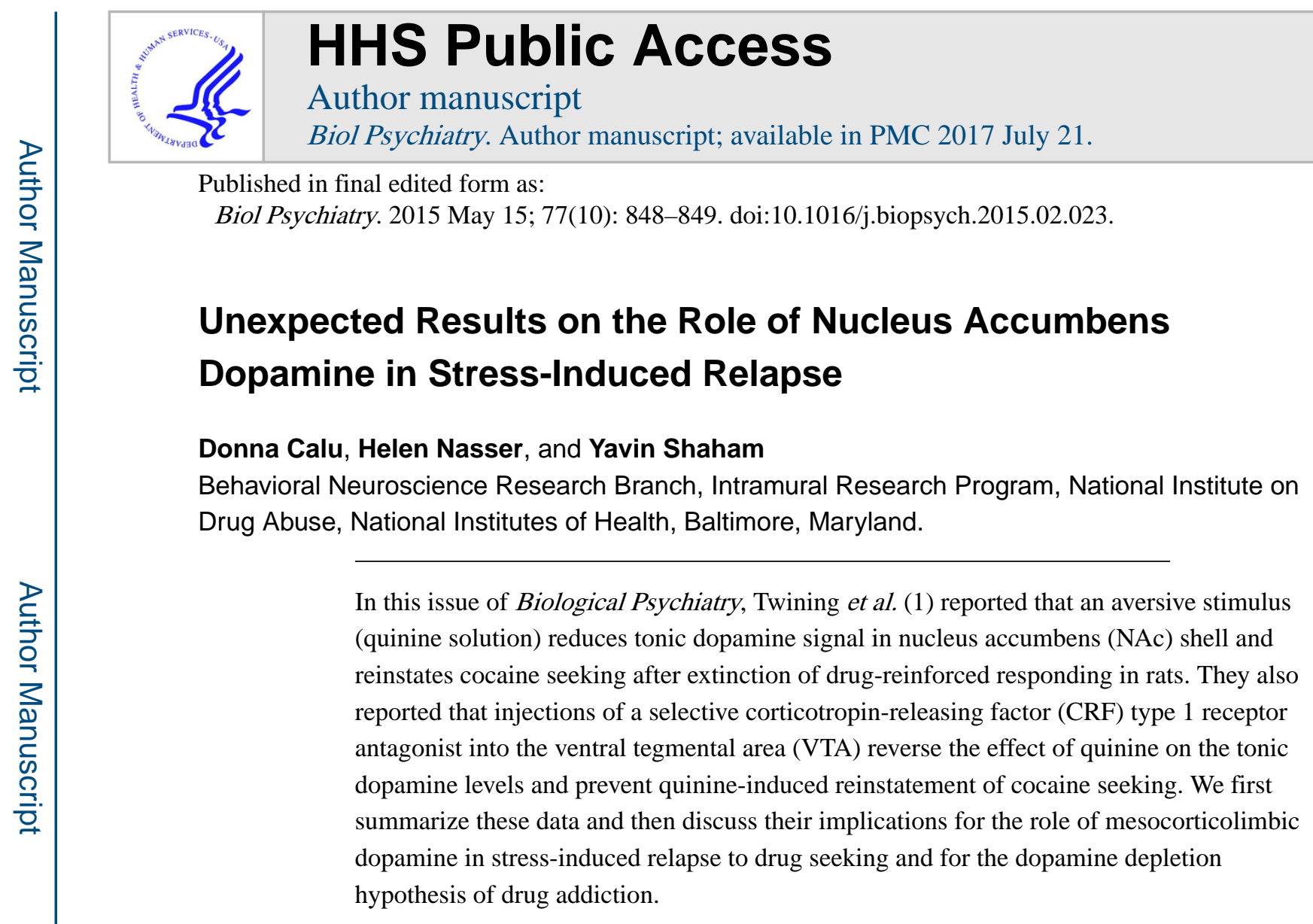

\title{
Summary of Study
}

The authors of this excellent study first used fast-scan cyclic voltammetry to monitor dopamine fluctuations in NAc shell during a session in which brief intraoral delivery of an aversive taste stimulus, a quinine solution, was passively delivered to freely moving drugnaïve rats. Quinine infusions induced characteristic aversive taste reactivity responses that were associated with time-locked (millisecond scale) reductions in phasic dopamine signaling in NAc. Intraoral quinine delivery also reduced NAc time-averaged dopamine concentrations that were evident at baseline and during the period after quinine delivery; this decrease was due to reduced dopamine transient frequency but not amplitude. The authors interpreted these data to indicate that quinine delivery caused a long-lasting (minute scale) decrease in tonic dopamine levels in NAc. Next, the authors used the reinstatement model to demonstrate that under identical quinine delivery parameters, the aversive taste stimulus reinstates drug seeking after extinction of drug-reinforced responding in rats with a history of intravenous cocaine self-administration. They also showed that blockade of CRF type 1 receptors in VTA of drug-naïve rats prevents quinine's effect on the reduction in tonic (but not phasic) dopamine concentrations in NAc and that the same manipulation in cocaineexperienced rats prevents quinine-induced reinstatement of cocaine seeking.

Address correspondence to Yavin Shaham, Ph.D., Behavioral Neuroscience Research Branch, Intramural Research Program, National Institute on Drug Abuse, National Institutes of Health, 251 Bayview Boulevard, Suite 200, Baltimore, MD 21224; yavin.shaham@nih.gov.

Disclosures

The authors report no biomedical financial interests or potential conflicts of interest. 


\section{Implications for the Role of Mesocorticolimbic Dopamine in Stress-Induced Relapse}

The present data are elegant and internally consistent, but they are highly unexpected based on the previous literature. In particular, since the early to mid 1980s, many studies using the reinstatement model indicate that reinstatement induced by aversive (intermittent footshock stress) and appetitive (drug cues or priming) stimuli is mediated by activation rather than inhibition of the mesocorticolimbic dopamine system (2). Additionally, the effect of intermittent footshock (a stressor that increases tonic dopamine concentrations in NAc) on reinstatement of cocaine seeking is prevented by blockade of CRF receptors in VTA $(3,4)$.

What might account for the seemingly contradictory results from the study by Twining et al.? One possibility is that tonic dopamine concentrations measured in fast-scan cyclic voltammetry are different from the tonic dopamine that is measured by in vivo microdialysis. We believe it is unlikely that the two methods would yield opposite changes in dopamine concentrations under the same experimental conditions because consistent with the finding by Twining et al. of reduced NAc dopamine by an aversive tastant is evidence from an early microdialysis study that taste aversion is associated with a rapid decrease in NAc dopamine concentrations (5). However, one issue to consider in interpreting the data of Twining et al. (1) is that, as mentioned earlier, the effect of quinine on NAc dopamine concentrations was determined in drug-naïve rats, whereas the effect of quinine on reinstatement of drug seeking was determined in cocaine-experienced rats. Particularly in reference to stress or aversive stimuli effects on mesocorticolimbic dopamine transmission, this difference may be critical. Wang et al. (3) reported that in cocaine-experienced (but not drug-naïve) rats, footshock stress increases CRF levels in VTA, leading to increased local glutamate release, which causes activation of the mesocorticolimbic dopamine system that leads to reinstatement of cocaine seeking.

Based on these findings, it remains an open question whether the aversive quinine taste would decrease NAc dopamine tone in drug-experienced rats in the presence of cocaineassociated contexts and cues and whether quinine-induced changes in tonic dopamine can directly drive cocaine seeking in these rats. Figure 1 illustrates the opposite effects that footshock and quinine stressors have on NAc dopamine concentrations, despite both changes being associated with increased cocaine seeking, and further illustrates the surprising conclusion from the study by Twining et al. and the above-described studies that CRF antagonism in the VTA regulates reinstatement induced by both stressors.

\section{Implications for the Dopamine Depletion Hypothesis}

The 1985 dopamine depletion hypothesis posits that cocaine addiction results from a withdrawal-induced dysphoric state in which the depletion of synaptic dopamine drives cocaine seeking to return dopamine to normal, drug-naïve levels (6). A series of in vivo microdialysis and electrical intracranial self-stimulation (a dopamine-dependent phenomenon) studies during the early 1990s provided a physiological basis for this hypothesis by demonstrating that during early withdrawal from different drugs of abuse the threshold for intracranial self-stimulation is increased (reflecting decreased brain stimulation 
reward) and extracellular dopamine levels in NAc are decreased $(7,8)$. However, with one potential recent exception (9), over the years investigators were unable to demonstrate in animal models of drug addiction that a NAc dopamine-depleted withdrawal state can motivate drug-taking behavior. For example, a study using the reinstatement model showed that naloxone-precipitated heroin withdrawal decreases NAc dopamine levels (assessed by in vivo microdialysis) but does not reinstate heroin seeking in opiate-dependent rats. In contrast, a spontaneous withdrawal condition, which does not decrease NAc dopamine levels, reinstates heroin seeking in the same rats (10).

In conclusion, from the perspective of the dopamine depletion hypothesis, the results of Twining et al. (1) are highly significant because they potentially provide the first evidence that a decrease in NAc dopamine tone, which has long been associated with the emergence of early drug withdrawal symptoms, can provoke relapse to drug seeking during abstinence. Although the authors' results are in line with the dopamine depletion hypothesis, it is surprising that such a finding has come from a study in which rats were trained under limited daily drug access conditions ( 2 hours/day) that do not lead to dependence-related symptoms in animal models of drug addiction, such as escalation of drug self-administration and decreases in brain stimulation reward (7).

\section{Acknowledgments}

This work was supported by the National Institute on Drug Abuse, Intramural Research Program.

\section{References}

1. Twining RC, Wheeler DS, Ebben AL, Jacobsen AJ, Robble MA, Mantsch JR, et al. Aversive stimuli drive drug seeking in a state of low dopamine tone. Biol Psychiatry. 2015; 77:895-902. [PubMed: 25442790]

2. Bossert JM, Marchant NJ, Calu DJ, Shaham Y. The reinstatement model of drug relapse: Recent neurobiological findings, emerging research topics, and translational research. Psychopharmacology (Berl). 2013; 229:453-476. [PubMed: 23685858]

3. Wang B, Shaham Y, Zitzman D, Azari S, Wise RA, You ZB. Cocaine experience establishes control of midbrain glutamate and dopamine by corticotropin-releasing factor: A role in stress-induced relapse to drug seeking. J Neurosci. 2005; 25:5389-5396. [PubMed: 15930388]

4. Blacktop JM, Seubert C, Baker DA, Ferda N, Lee G, Graf EN, et al. Augmented cocaine seeking in response to stress or CRF delivered into the ventral tegmental area following long-access selfadministration is mediated by CRF receptor type 1 but not CRF receptor type 2. J Neurosci. 2011; 31:11396-11403. [PubMed: 21813699]

5. Mark GP, Blander DS, Hoebel BG. A conditioned stimulus decreases extracellular dopamine in the nucleus accumbens after the development of a learned taste aversion. Brain Res. 1991; 551:308310. [PubMed: 1913157]

6. Dackis CA, Gold MS. New concepts in cocaine addiction: The dopamine depletion hypothesis. Neurosci Biobehav Rev. 1985; 9:469-477. [PubMed: 2999657]

7. Markou A, Koob GF. Postcocaine anhedonia: An animal model of cocaine withdrawal. Neuropsychopharmacology. 1991; 4:17-26. [PubMed: 2003866]

8. Rossetti ZL, Hmaidan Y, Gessa GL. Marked inhibition of mesolimbic dopamine release: A common feature of ethanol, morphine, cocaine and amphetamine abstinence in rats. Eur J Pharmacol. 1992; 221:227-234. [PubMed: 1426002]

9. Willuhn I, Burgeno LM, Groblewski PA, Phillips PE. Excessive cocaine use results from decreased phasic dopamine signaling in the striatum. Nat Neurosci. 2014; 17:704-709. [PubMed: 24705184] 
10. Shaham Y, Rajabi H, Stewart J. Relapse to heroin-seeking under opioid maintenance: The effects of opioid withdrawal, heroin priming and stress. J Neurosci. 1996; 16:1957-1963. [PubMed: 8774462] 


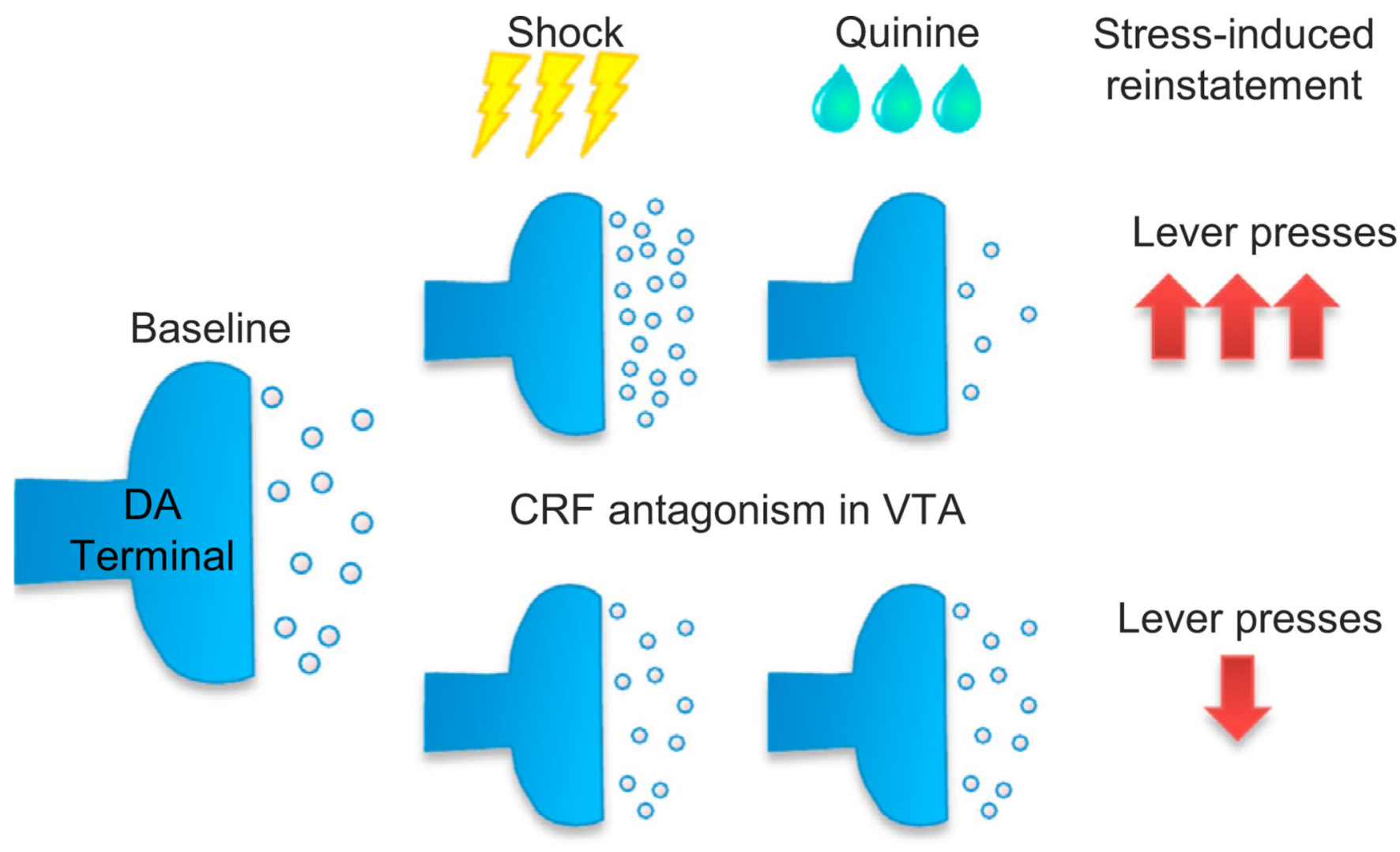

Figure 1.

The effects of intermittent footshock stress and aversive quinine solution on extracellular dopamine concentrations in nucleus accumbens and on reinstatement of cocaine seeking. The two aversive stimuli cause reinstatement of cocaine seeking but have opposite effects on dopamine release from the mesolimbic dopamine system (the ventral tegmental area dopamine neurons that project to the nucleus accumbens). Unexpectedly, blockade of corticotropin-releasing factor receptors in the ventral tegmental area blocks the effect of stressors on reinstatement of cocaine seeking and activation (footshock) or inhibition (quinine) of this dopamine system. Data summarize results from the study by Twining et al. (1) and previous published reports $(3,4)$. CRF, corticotropin-releasing factor; DA, dopamine; VTA, ventral tegmental area. 